\title{
Direction of Arrival Tracking by Adaptive Subspace Methods
}

\author{
Khairy ElBarbary ${ }^{*}$, Tarek Baheron ${ }^{* *}$
}

\begin{abstract}
The radar tracking system prefers to have an estimated covariance matrix in a single snapshot in order to estimate the next position of a moving target. Recent research provides the inflation method and the projection approximation subspace tracking (PAST) method to estimate the DOA based on successive snap shots where an objective function, such as minimize the DOA estimation error, is defined and minimized. In this paper we provide complete analysis in addition to computer simulations for the mentioned recently published DOA estimation methods. Their behaviors are compared with the traditional, parametric, Eigen decomposition and spatial smoothing methods. Although the spatial smoothing method outperforms the other traditional method, that it could estimate the DOA of both coherent and non coherent signals at higher SNR. The recent DOA estimation method provided the capability of tracking a moving source as well as estimating the DOA of fixed location source. PAST method, can track moving signal sources, which change their direction.
\end{abstract}

\section{Introduction}

The subspace methods such as MUSIC or ESPRIT, for direction of arrival (DOA) estimation, are based on the Eigen structure of the estimated covariance matrix of the data received by antenna array. As the number of the array snapshots increases as the accuracy of the estimated covariance matrix increases, consequently the accuracy of the estimated DOA. However, these methods are still too complicated to admit real-time implementations and they are unsuitable for non-stationary environments. In many applications, such as tracking of a moving source of electromagnetic radiation it is required to estimate the $D O A$ for every new snapshot. Therefore, adaptive subspace-based methods are introduced to overcome this drawback and to alleviate the computational overhead for fast subspace tracking. The adaptive Pisarenko's Harmonic Retrieval (PHR) method, proposed by Thompson [1], utilizes an adaptive method such as least mean-square (LMS) to obtain a set of minimum eigenvectors to estimate the noise subspace and consequently the DOAs of the array impinging signals. The Gauss-Newton iterations [2] [3], and conjugate gradient methods [4] can also be applied to seek the largest or smallest eigenvalues and their corresponding eigenvectors adaptively. Rank revealing $U R V$ decomposition $[5,6]$ is proposed to track the signal or noise subspace. Yang et al proposed an inflation method which combines many adaptive PHRs for tracking of the noise subspace [7]. Besides, a general inflation method was also addressed to overcome the setbacks of the original inflation method [8]. On the other hand, a projection approximation subspace tracking (PAST) method, based on recursive-least-square (RLS) method via a novel interpretation of the projection as an unconstrained optimization problem was considered in [9]. The tracked subspace is then employed by the MUSIC or ESPRIT to locate the DOAs of the array impinging signals.

\footnotetext{
* Egyptian Armed Forces

** PGS student.
} 
This paper provides analytic evaluation of the proposed methods for subspace tracking as well as computer simulation for their behavior for different practical situations. Sections II and III provide brief description as well as analytic derivation of the inflation method and PAST method for signal or noise subspace tracking respectively. Section IV provides computer simulation for the behavior of the inflation and PAST methods for noise subspace tracking and the utilization of MUSIC method for $D O A s$ estimation based on the tracked noise subspace. Finally section $\mathrm{V}$ provides conclusions and recommendations for future work.

\section{Inflation Method for DOA Estimation}

The subspace-based methods such as MUSIC and ESPRIT are based on batch estimation of the data covariance matrix. This estimation requires a large number of consequent array snapshots which makes the subspace based method unsuitable for non-stationary environments. Therefore, adaptive subspace-based methods are introduced to overcome this drawback. In this paper, some DOA tracking methods are analyzed and evaluated through computer simulation. Namely, the inflation method [7], and the PAST method [9, 10] are considered. Yang et al [7] proposed a highly parallel method, denoted by the inflation method, for the estimation of the signal or noise subspace of the correlation matrix of the received data by an adaptive array. The general formulation of the inflation method results from an asymptotic argument which shows the signal or noise subspace computation to be a constrained searching procedure. The basic structure of the adaptive Pisarenko's Harmonic Retrieval (PHR) is illustrated in Fig.1 [1]. It is consists of L elements antenna array followed by adaptive weights vector. An adaptive subspace based methods such as the least mean square, LMS and the recursive least squares, RLS [11] are used to obtain the optimum weights by an iterative procedure. These optimum weights vector will converge to the signal or noise subspace. The detailed structure of the adaptive Eigen-subspace Linear Combiners for direction of arrival estimation is shown in Fig.2.

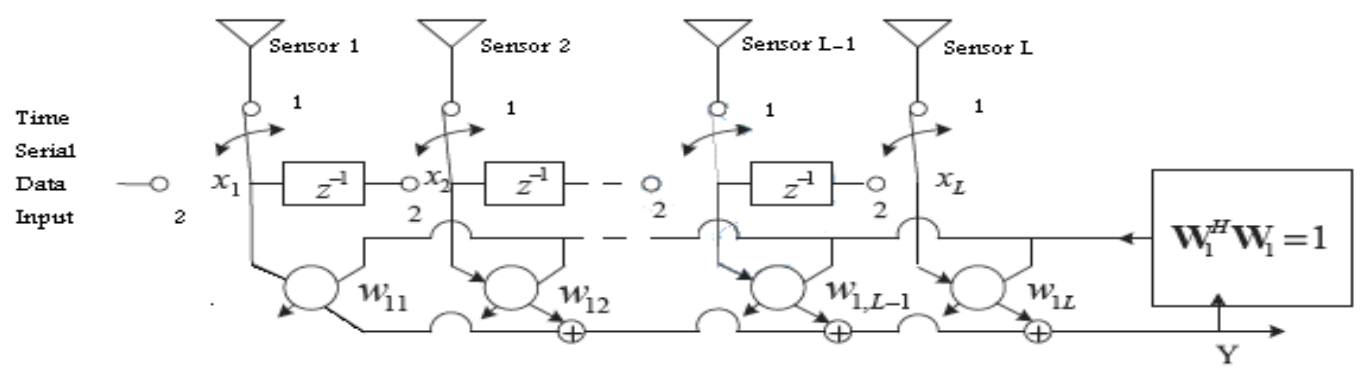

Fig.1. Structure of the adaptive Pisarenko’s Harmonic Retrieval

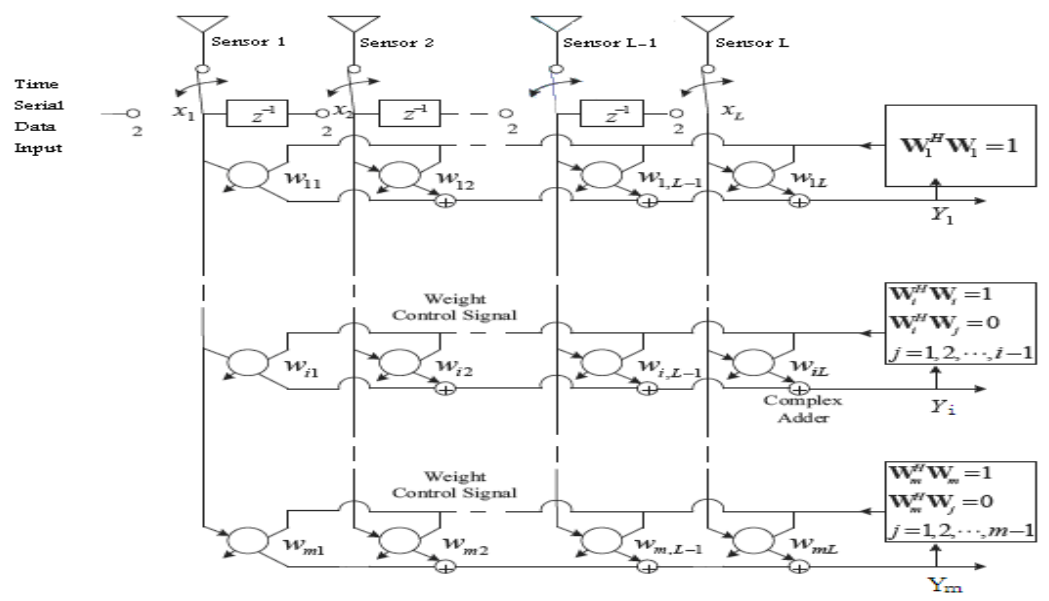

Fig.2. Adaptive Eigen-subspace Linear Combiners 
The matrix of the adaptive weights are considered as m- identical independent linear combiners each combiner is an orthonormal set of weights, which is given by

$$
W_{i}=\left[w_{i 1}, w_{i 1}, \cdots, w_{i_{L}}\right]^{T}, \quad i=1,2, \cdots, m
$$

For stationary data, the output of the $i^{\text {th }}$ linear combiner is

$$
y_{i}(t)=W_{i}^{H} X(t), \quad i=1,2, \cdots, m
$$

The number of combiners, $\mathrm{m}$, is chosen to be equal to the estimated number of signals impinging the array, $\mathrm{M}$ which determines the dimension of the signal subspace. Conversely, it may be chosen as $m=L-M$, for estimation of the noise subspace.

In stationary situation, the desired steady-state weights could be obtained by minimizing a cost function with respect to the signal subspace or maximizing it with respect to the noise subspace estimation. The cost function is defined as the average power of the linear combiners output and it is given by

$$
J=E\left[Y^{H} Y\right]=E\left[\operatorname{tr}\left(y y^{H}\right)\right]=E\left[\operatorname{tr}\left(W^{H} X X^{H} W\right)\right]=\operatorname{tr}\left(W^{H} R W\right)
$$

Minimization of cost function $J$ could be accomplished by applying the constrained gradient-search procedure. If the convergence rate $\mu$ is constant, then, the weight matrix is updated by [7].

$$
W^{\prime}(t)=W(t-1)-\mu \nabla(t)
$$

Where, $W(t)$ is obtained by taking the Gram-Schmidt orthogonalization of the columns of $W^{\prime}(t)$ and $\mu$ is a small positive constant. $\nabla(t)$ is an estimator of the gradient of $J$ with respect to $W$, which is approximated by $X(k) y_{i}(k)$.

The update equations of the inflation method can be summarized as follows:

$$
\begin{gathered}
X_{i}(k)=X_{i-1}(k)+W_{i-1}(k) y_{i-1}(k) \quad i=2,3, \ldots, L-M \\
X_{1}(k)=X(k) \\
y_{i}(k)=W_{i}^{H} X_{i}(k) \quad i=1,2, \ldots, L-M \\
W_{i}^{\prime}(k)=W_{i}(k-1)-2 \mu X(k) y_{i}(k) \\
W_{i}(k)=\frac{W_{i}^{\prime}(k)}{\left[W_{i}^{\prime H}(k) W_{i}^{\prime}(k)\right]^{\frac{1}{2}}}
\end{gathered}
$$

A generalized inflation method is proposed in [8], where the updated data vector can be expressed as

$$
\begin{gathered}
X_{i}(k)=X_{i-1}(k)+\alpha W_{i-1}(k) y_{i-1}(k) \quad i=2,3, \ldots, L-M \\
X_{1}(k)=X(k)
\end{gathered}
$$

Where, $\alpha$ is a forgetting factor which is ranged as $0<\alpha<1$.

\section{A projection Approximation Subspace Tracking (PAST) method.}

A projection Approximation Subspace Tracking (PAST) method [9, 12] is based on a novel interpretation of the signal subspace as the solution of a projection like unconstrained minimization problem. The unconstrained minimization can be solved by RLS [11, 13] techniques by making an appropriate projection approximation. More specifically, based on the data model, we first consider an unconstrained cost function given by

$$
J(W)=E\left\{\left\|X-W W{ }^{H} X\right\|^{2}\right\}=\operatorname{tr}(R)-2 \operatorname{tr}\left(W^{H} R W\right)+\operatorname{tr}\left(W^{H} R W \cdot W^{H} W\right)
$$


With the corresponding correlation matrix, $R_{X}=E\left[X X{ }^{H}\right]$. We can approximate (11) by replacing the involved expectation with an exponentially weighted sum to yield

$$
\begin{gathered}
J(W(t))=\sum \beta^{t-1}\left\|X(i)-W(t) W^{H}(t) X(i)\right\|^{2} \\
=\operatorname{tr}[R(t)]-2 \operatorname{tr}\left[W^{H}(t) R(t) W(t)\right]+\operatorname{tr}\left[W^{H}(t) R(t) W(t) W^{H}(t) W(t)\right]
\end{gathered}
$$

Where, $1 \leq i \leq t$ and the forgetting factor is $0 \leq \beta \leq 1$.

Note that $J(W(t))$ in (12) is identical to $J(W)$ in (11) except for the use of the exponentially weighted correlation matrix, defined as

$$
R(t)=\sum_{i=1}^{t} \beta^{t-i} X(i) X^{H}(i)=\beta R(t-1)+X(t) X^{H}(t)
$$

to replace the original correlation matrix R. The main issue of the PAST method is to approximate $W^{H}(t) X(i)$ in (12), which is the unknown projection of $X(i)$ onto the columns of $W(t)$ by $Y(i)=W^{H}(i-1) X(i)$. Therefore, we can obtain the modified cost function given by

$$
J^{\prime}(W(t))=\sum_{i=1}^{t} \beta^{t-i}\|X(i)-W(t) Y(i)\|^{2}
$$

Which is quadratic in the elements of $W(t)$.

We expect $J^{\prime}(W(t))$ to be a good approximation for $J(W(t))$ and the matrix $W(t)$ minimizing $J^{\prime}(W(t))$ to be a good estimate for the signal subspace of $R(t)$. The main advantage of the PAST method is the exponentially weighted least squares criterion in (14), which can be solved by applying various RLS methods [11]. Therefore, the modified cost function $J^{\prime}(W(t))$ is minimized if

$$
\begin{gathered}
W(t)=R_{x y}(t) R_{y y}^{-1}(t) \\
R_{x y}(t)=\sum_{i=1}^{t} \beta^{t-1} X(i) Y^{H}(i)=\beta R_{x y}(t-1)+X Y^{H}(t) \\
R_{y y}(t)=\sum_{i=1}^{t} \beta^{t-1} Y(i) Y^{H}(i)=\beta R_{y y}(t-1)+Y Y^{H}(t)
\end{gathered}
$$

Note that a more efficient and numerically more robust method is to apply the matrix inversion lemma to compute the inverse of $R_{y y}(t)$ \{denoted as $P(t)$ \} or to use the Q-R updating technique to calculate the Cholesky factor of $R_{y y}(t)$ recursively [11], that

$$
\begin{aligned}
& \text { Choose } P(0) \text { and } W(0) \text { suitably } \\
& \text { FOR } t=1,2, \ldots \text { DO } \\
& Y(t)=W^{H}(t-1) X(t) \\
& h(t)=P(t-1) Y(t) \\
& g(t)=h(t) /\left[\beta+Y^{H} h(t)\right] \\
& P(t)=\frac{1}{\beta} \operatorname{Tri}\left\{P(t-1)-g(t) h^{H}(t)\right\} \\
& e(t)=X(t)-W(t-1) Y(t) \\
& W(t)=W(t-1)+e(t) g^{H}(t)
\end{aligned}
$$

These above equations summarize the PAST method for tracking the signal subspace. The operator Tri(.) indicates that only the upper (or lower) triangular part of $P(t)=R_{y y}^{-1}$ is calculated and its Hermitian transposed version is copied to the other lower (or upper) triangular part. This RLS scheme reduces the number of operations and preserves the Hermitian symmetry of $P(t)$ in presence of 
rounding errors. The initial values $P(0)$ and $W(0)$ have to be chosen suitably. $P(0)$ has to be a Hermitian positive definite matrix. $W(0)$ should contain $M$ orthonormal vectors. However, we usually set $P(0)$ to the $M \times M$ identity matrix and the columns of $W(0)$ to the $M$ leading unit vectors of the $L \times L$ identity matrix.

\section{Simulation Results.}

\section{IV.1. Simulation of Inflation Method}

As an illustration, an example is conducted to evaluate the inflation tracking method. The number of antennas elements in the linear array is assumed to be $L=9$ and 256 successive snapshots are utilized and the convergence factor $\mu$ is set as $2.5 \times 10^{-5}$. Fig. 3 shows the tracking curves for two stationary signal sources with fixed and distinct DOAs $\left\{-10^{\circ}, 10^{\circ}\right\}$ where the SNR is set to be $6 d B$ and $20 d B$ in Fig. 3 (a), (b) respectively. Also, Fig. 4 shows the tracking curves of two stationary signal sources with closely spaced DOA`s $\left\{-5^{\circ}, 5^{\circ}\right\}$ where the SNR is set to be $6 d B$ and $20 d B$ in Fig.4 (a), (b) respectively. As we can observe from Fig.3, and Fig.4, the DOA tracking curve will converge, after about 200 iterations for distinct separated sources, $\left\{-10^{\circ}, 10^{\circ}\right\}$ for low SNR $=6 \mathrm{~dB}$. Also it will converge at about 250 iterations for closely spaced sources, $\left\{-5^{\circ}, 5^{\circ}\right\}$ for low SNR $=6 \mathrm{~dB}$. Finally increasing the SNR to $20 \mathrm{~dB}$ fasts the convergence of the inflation method for both cases of distinct and closely spaced sources.

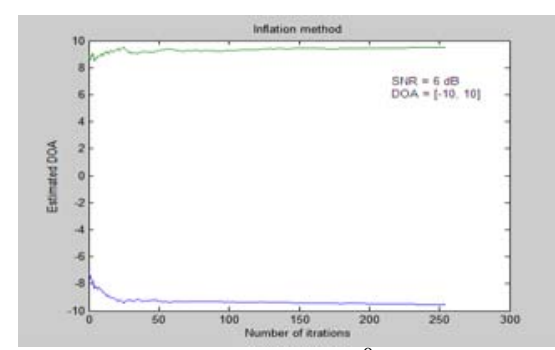

(a) $\mathrm{DOA}=\left\{-10^{\circ}, 10^{\circ}\right\}, \mathrm{SNR}=6 \mathrm{~dB}$

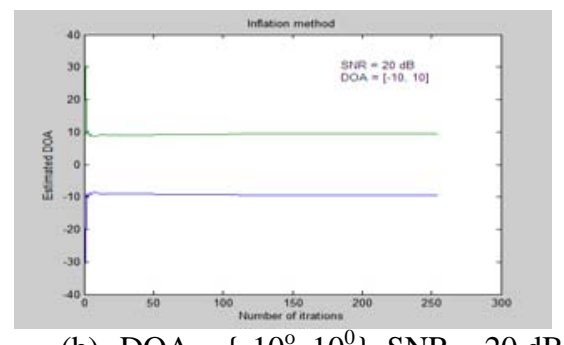

(b) $\mathrm{DOA}=\left\{-10^{\circ}, 10^{\circ}\right\}, \mathrm{SNR}=20 \mathrm{~dB}$

Fig.3. Performance of inflation method for distinct spaced signal sources.

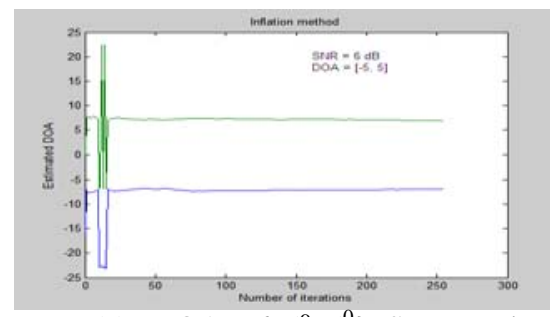

(a) $\mathrm{DOA}=\left\{-5^{\circ}, 5^{0}\right\}, \mathrm{SNR}=6 \mathrm{~dB}$

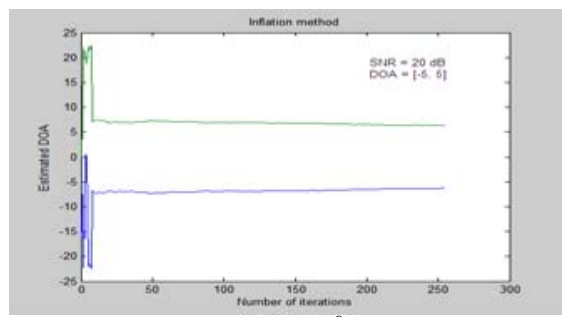

(b) $\mathrm{DOA}=\left\{-5^{0}, 5^{0}\right\}, \mathrm{SNR}=20 \mathrm{~dB}$

Fig.4. Performance of inflation method for closely spaced signal sources.

\section{IV.2. Simulation of the PAST Method}

As an illustration, the same example is conducted to evaluate the PAST method. The SNR is set to be $6 \mathrm{~dB}$ and $20 \mathrm{~dB}$, the number of array elements is assumed to be 9 elements and the number of considered snapshot is 256 snapshots. The forgetting factor $\beta$ is set to equal $2.5 \times 10^{-5}$. The tracking curve for estimating the DOA of two moving signals sources 
start at DOAs $\left\{-10^{\circ}, 10^{\circ}\right\}$ and approach each other are shown in Fig.5 (a), (b) for SNR $=6 \mathrm{~dB}$ and $20 \mathrm{~dB}$ respectively. The tracking curve for a very close space moving signals sources start at DOAs $\left\{-5^{\circ}, 5^{\circ}\right\}$ and approach each other are shown in Fig.6 (a), (b) for SNR $=6 \mathrm{~dB}$ and $20 \mathrm{~dB}$ respectively. It is clear from Fig. 6 , that the PAST method, will estimate the true DOA of two signals starts at $\left\{-5^{\circ}, 5^{\circ}\right\}$, after 100 iterations, for low SNR as in Fig. 6 (a), while the true DOA of two closely spaced signals is estimated after 20 iterations for high SNR as in Fig. 6 (b). However in case of distinct, approaching sources the convergence is faster as in Fig. 5.

Fig.7, presents the performance evaluate of the PAST method in estimating the DOA of one fixed at $\left\{-8^{0}\right\}$ and two moving targets start at DOAs $\left\{8^{\circ}, 12^{\circ}\right\}$ and approach each other. The performance is evaluated for different $\mathrm{SNR}=6 \mathrm{~dB}, 20 \mathrm{~dB}$. It is clear from Fig. 7, that the PAST method successfully determine the DOA of the three sources $(-8,12,8)$ after 50 iterations. Finally comparing Fig. 7 (a), (b) we can note that increasing of SNR fasts the convergence of the PAST Method.

\section{Conclusions}

The inflation tracking algorithm, is capable to track stationary signal sources with fixed and closely spaced DOA `s. Moreover, increase of SNR fasts the convergence of the inflation method. PAST algorithm, can track moving signal sources, which change their direction. Increase of SNR fasts the convergence of the PAST Algorithm and provide accurate DOA estimation. It is recommended to study the required modifications to both analyzed algorithms in order to operate and cope with modern wide band communications signals.

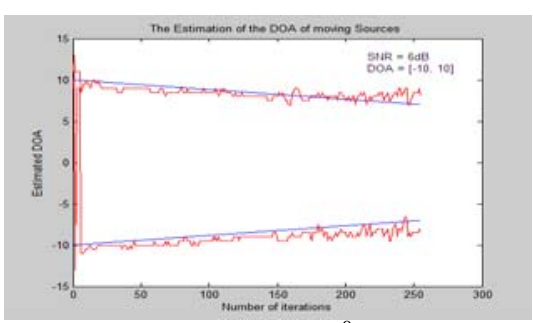

(a) $\mathrm{DOA}=\left\{-10^{\circ}, 10^{\circ}\right\}, \mathrm{SNR}=6 \mathrm{~dB}$

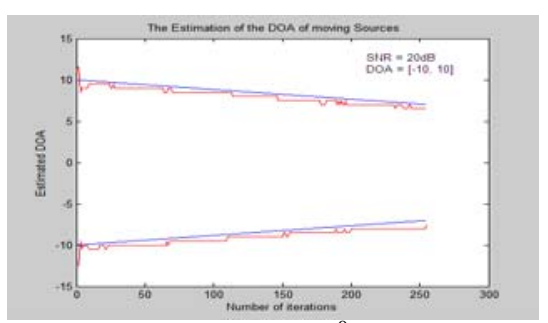

(b) $\mathrm{DOA}=\left\{-10^{\circ}, 10^{\circ}\right\}, \mathrm{SNR}=20 \mathrm{~dB}$

Fig.5. Performance of the PAST method for estimation the DOA of distinct moving sources.

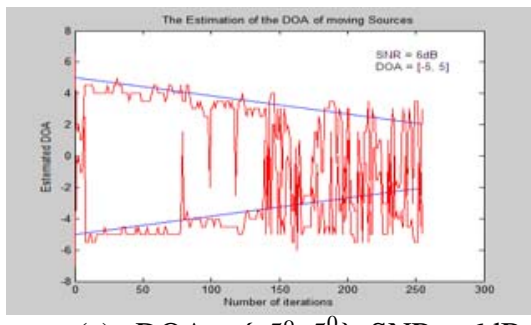

(a) $\mathrm{DOA}=\left\{-5^{\circ}, 5^{0}\right\}, \mathrm{SNR}=6 \mathrm{~dB}$

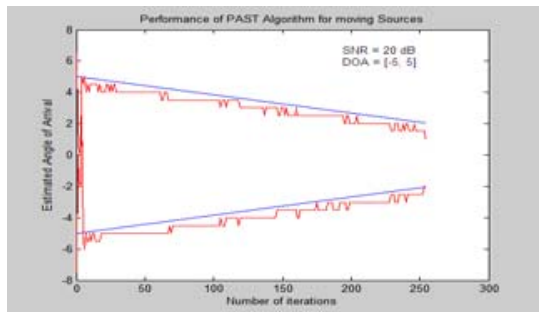

(b) $\mathrm{DOA}=\left\{-5^{0}, 5^{0}\right\}, \mathrm{SNR}=20 \mathrm{~dB}$

Fig.6. Performance of the PAST method for estimation the DOA of closely moving sources. 


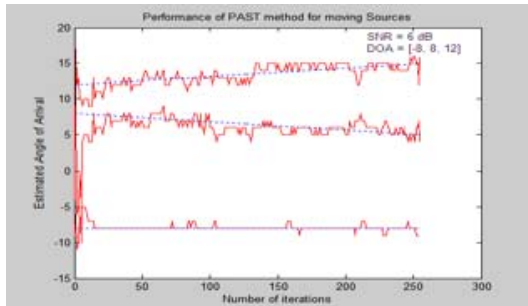

(a) $\mathrm{DOA}=\left\{-8^{\circ}, 8^{0}, 12^{0}\right\}, \mathrm{SNR}=6 \mathrm{~dB}$

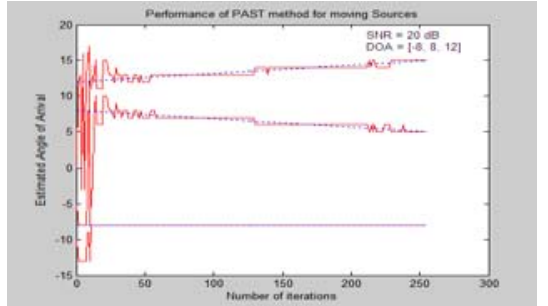

(b) $\mathrm{DOA}=\left\{-8^{0}, 8^{0}, 12^{0}\right\}, \mathrm{SNR}=20 \mathrm{~dB}$

Fig.7. Performance evaluation of the PAST method for estimation the DOA of two moving and one fixed sources

\section{References}

[1] P. A. Thompson, "An adaptive spectral analysis technique for unbiased frequency estimation in the presence of the white noise," in Proc. $13^{\text {th }}$ Asilomar Conf. Circuit, Syst. Comput., Pacific Grove, CA, 1980.

[2] V.U. Reddy, B. Egardt and T. Kailath, "Least squares type algorithm for adaptive implementation of Pisarenko's Harmonic retrieval method,” IEEE Trans. Acoustic, Speech, Signal Processing, vol. ASSP-30, 1982.

[3] S. Bannour and M. R. Azimi-Sadjadi, "An adaptive approach for optimal data reduction using recursive least squares learning method," in Proc. IEEE ICASSP pp. II297-II300 San Francisco, CA, 1992.

[4] X. Yang, T. K. Sarkar and E. Arvas, "A survey of conjugate gradient algorithms for solution of extreme Eigen-problems of a symmetric matrix," IEEE Trans. Acoustic., Speech, Signal Processing, vol. 37, pp. 1550-1556, 1989.

[5] G. W. Stewart, "An updating algorithm for subspace tracking," IEEE Trans, Signal Processing, vol. 40, pp. 1535-1541, 1992.

[6] C. H. Bischof and G. M. Shroff, “On updating signal subspace,” IEEE Trans, Signal processing, vol. 40, pp. 96-105, 1992.

[7] J.-F Yang and M. Kaveh, "Adaptive Eigen subspace algorithms for direction or frequency estimation and tracking," IEEE Trans. on Acoustics, Speech, and Signal Processing, pp. 241-251, Feb. 1988.

[8] J.-F Yang and H.-J. Lin, "Adaptive high-resolution algorithms for tracking nonstationary sources without the estimation of source number," IEEE Trans. Signal Processing, vol. 42, pp. 563-571, March 1994.

[9] B. Yang, "Projection approximation subspace tracking," IEEE Trans. On Signal Processing, pp. 95-107, Sept. 1995. invariance techniques," IEEE Tran. ASSP, vol. 37, pp. 984-995, July 1989.

[10] W.D. Blair, B.J. Slocumb, G.C. Brown and A.H. Register, "Tracking of closely spaced, possibly unresolved, Rayleigh targets idealized resolution,” in Proc. IEEE Aerospace Conf., pp. 1543-1550, 2002.

[11] S. Haykin, Adaptive Filter Theory, $3^{\text {rd }}$ ed, Prentice-Hall, New Jersey, 1996.

[12] B. He, D. Wang, M. Phain and T. Yu, "Position and orientation estimation with high accuracy for a car-like vehicle,” in Proc. IEEE Intelligent Transportation Systems Conf., pp. 528-533, 2002.

[13] A. Kuchar, M. Tangemann and E. Bonek, "A real-time DOA-based smart antenna processor,” IEEE Trans. on Vehicular Technology, pp. 1279- 1293, 2002. 